\title{
Plantarflexor and Dorsiflexor Activation during Inclined Walking with and without Modified Mobilization with Movement Using Tape in Women with Limited Ankle Dorsiflexion
}

\author{
J-Yeon Yoon, PT, MS ${ }^{1)}$, Duk-Hyun An, PT, PhD²), JAe-Seop Oh, PT, PhD ${ }^{2)}$ \\ 1) Department of Rehabilitation Science, Graduate School, Inje University \\ 2) Department of Physical Therapy, College of Biomedical Science and Engineering, Inje University: \\ 607 Obang-dong, Gimhae, Gyeongsangnam-do 621-740, South Korea. TEL: +82 55-320-3680, \\ FAX: +82 55-329-1678
}

\begin{abstract}
Purpose] This study compared the EMG activities of the plantarflexor and dorsiflexor muscles during inclined walking with and without modified mobilization with movement (modified MWM) using tape in women with limited ankle dorsiflexion. [Subjects] Fifteen women with limited dorsiflexion in their feet (22 feet in total) were recruited for this study. [Methods] The subjects walked with and without modified MWM using tape on a treadmill at 6 degrees with a speed of $1.25 \mathrm{~m} / \mathrm{s}$ for 5 minutes. The EMG activities of the medial gastrocnemius (GCM) and tibialis anterior (TA) muscles were measured using a surface EMG system. [Results] During incline walking with modified MWM using tape, the mean EMG activity of the GCM significantly increased, and that of the TA decreased between heel strike and heel off. There was no difference between heel off and toe off in the two muscles. [Conclusions] Modified MWM using tape on the talus during incline walking could alter the muscle activities of the GCM and TA between heel strike and heel off in women with limited ankle dorsiflexion.

Key words: Ankle dorsiflexion, Inclined walking, Tape
\end{abstract}

(This article was submitted Mar. 13, 2013, and was accepted Apr. 2, 2013)

\section{INTRODUCTION}

Plantarflexor muscles play a critical role in ankle stabilization, and their combined eccentric contraction decreases the rate of tibial advancement during midstance and causes the foot and tibia to roll off over the forefoot rocker during terminal stance ${ }^{1)}$. Limited ankle dorsiflexion range of motion (ROM) is associated with various cumulative injuries and also causes compensatory changes during walking $^{2}$. For normal walking, proper ankle dorsiflexion ROM is necessary to absorb the body weight and contributes to the forward body movement during the stance phase of the gait cycle ${ }^{1)}$. Ambulation on a sloped surface requires a shift towards increased ankle dorsiflexion and more muscle activation of the knee and ankle joint for stability ${ }^{3}$. Deficits in ankle dorsiflexion ROM can come from restraints in contractile or noncontractile tissue, and abnormal tissue flexibility requires greater force, which leads to energy dissipation $^{1,4)}$. Therefore, several methods have been examined to increase ankle dorsiflexion ${ }^{5,6)}$. Mobilization with movement (MWM) is widely used to improve the accessory motion of joints; however, it has a limitation in that it can only be applied by a therapist in a physical therapy room ${ }^{7}$. Recently, MWM has been modified by using tape to pro-

*To whom correspondence should be addressed. E-mail: dhahn670208@gmail.com vide a posterior inferior talar glide, which can cause passive movement by substituting for a therapist's force ${ }^{8)}$. A lot of physical therapists have often employed MWM using tape to increase ankle dorsiflexion, but how incline walking with modified MWM using tape influences the foot and ankle biomechanics is still unclear, and no information has been gathered about the muscle activities. An inclined surface is often used as a therapeutic intervention because it requires additional muscle action and increases the concentric activity of the plantarflexor muscles ${ }^{9}$. Biomechanical alterations resulting from clinicians' utilization of modified MWM using tape have the potential to change the plantarflexor and dorsiflexor muscle activities. Therefore, the purpose of this study was to investigate the effect of modified MWM using tape on the talus on the GCM and TA muscle activities during incline treadmill walking in women with limited ankle dorsiflexion.

\section{SUBJECTS AND METHODS}

Fifteen women (a total of 22 feet with limited ankle dorsiflexion) were recruited for this study. The mean age, height, and weight of the subjects were $28.1 \pm 3.0$ years (mean $\pm \mathrm{SD}$ ), $161.9 \pm 6.9 \mathrm{~cm}$, and $54.5 \pm 5.0 \mathrm{~kg}$, respectively. Our eligibility criteria were as follows: 1) passive ankle dorsiflexion ROM of less than 8 degrees when measured in a non-weight-bearing condition with the knee extended 
and ankle dorsiflexion ROM with the knee flexed to at least 5 degrees greater than with the knee extended in the subtalar neutral position; 2) leg length discrepancy of less than $2 \mathrm{~cm} ; 3$ ) no lower extremity injury within 6 months prior to participation in the study; 4) no history of neurological dysfunction; and 5) ability to ambulate without pain. We chose experimental feet with a passive ankle dorsiflexion ROM of 8 degrees or less with the knee extended ${ }^{6}$.

A VICON MX-T20 motion analysis system (VICON Motion System, Ltd., Oxford, UK) (sampling rate $100 \mathrm{~Hz}$ ) with eight cameras was used to record the location of reflective skin markers over the lateral calcaneus; this was used to determine the time of heel strike and heel off. The activities of the medial GCM and TA muscles of the lower extremity with limited ankle dorsiflexion were measured by a wireless electromyography (EMG) system (Delsys, Inc., Boston, MA, USA) using surface electrodes with a fixed interelectrode distance of $10 \mathrm{~mm}$. The EMG signals were rectified and low-pass filtered using a zero-lag Butterworth filter with a cutoff frequency of $10 \mathrm{~Hz}$. The data processing was controlled by the Nexus software (ver. 1.4), and the EMG data were synchronized with the motion analysis recordings. Gait cycle events were used to determine heel strike, heel off, and toe off events for each gait trial. Prior to incline walking, electrodes were attached to the medial GCM and TA muscles. A sampling rate of $1,000 \mathrm{~Hz}$ was used, and the root mean square (RMS) values of the raw data were calculated.

For normalization, maximal EMG signals were acquired during a maximal voluntary isometric contraction (MVIC) maneuver, which was performed for 5 seconds in the manual muscle-testing position described by Kendall et al ${ }^{10)}$. Each subject was instructed to walk with and without modified MWM using tape on an inclined treadmill (model 1100, HEBB Industries, Inc., Tyler, Texas, USA) set to a grade of 6 degrees at a speed of $1.25 \mathrm{~m} / \mathrm{s}$ for 5 minutes. The EMG data were collected during incline walking in the last 30 seconds. The modified MWM using taping was applied by one examiner's maximal force using a piece of tape. Each participant placed their foot on a box and positioned closedpacked dorsiflexion as tibia forward from the talus anterior progressing inferiorly and posteriorly. One examiner attached the tape (a Mueller kinesiological tape with a width of $37 \mathrm{~mm}$ ) to the plantar surface of the calcaneus from the lateral to medial side. The two tasks (incline walking with and without modified MWM using tape) were administered randomly, and each subject rested for 30 minutes between tasks in order to minimize any learning effect. Statistical analysis was performed using a statistical package (PASW statistics, 18.0, IBM corporation, NY). The paired t-test was used to examine differences in EMG activities of the GCM and TA muscles with and without modified MWM using tape, and significance was defined as $\mathrm{p}<0.05$.

\section{RESULTS}

There were significant differences with and without the modified MWM using tape in terms of the normalized mean EMG activity of the GCM $(p=0.001)$ and TA $(p=0.049)$ between heel strike and heel off. The normalized mean EMG activities of the GCM during incline walking with and without the modified MWM using tape were $67.63 \pm 21.48 \% \mathrm{MVIC}$ and $54.56 \pm 17.68 \% \mathrm{MVIC}$, respectively. The normalized mean EMG activities of the TA during incline walking with and without the modified MWM using tape were $24.85 \pm 15.97 \% \mathrm{MVIC}$ and $27.52 \pm 15.14 \% \mathrm{MVIC}$, respectively. There were no significant differences among tasks in terms of the EMG activities of the GCM and TA between heel off and toe off. The normalized mean EMG activities of the GCM during incline walking with and without the modified MWM using tape were $56.24 \pm 29.79 \%$ MVIC and $52.56 \pm 19.05 \% \mathrm{MVIC}$, respectively. The normalized mean EMG activities of the TA during incline walking with and without the modified MWM using tape were $26.86 \pm 16.48 \%$ MVIC and $24.97 \pm 12.54 \%$ MVIC, respectively.

\section{DISCUSSION}

This study investigated the effect of modified MWM using tape on muscle activities during inclined walking in women with limited ankle dorsiflexion. We found that modified MWM using tape during incline walking increased the activity of the medial GCM muscle and decreased the activity of the TA muscle between heel strike and heel off, while there was no difference between heel off and toe off in the two muscles. We consider that applying tape to the talus during incline walking may make the talus move in the posterior and inferior directions. It is also possible that the force of the tape toward the posterior and inferior directions on the talus and a repetitive activity involving 5 minutes of incline walking performed additively could induce the talus to roll and glide posteriorly, which may affect ankle arthrokinematics, resulting in a gain in the normal dorsiflexion ROM. Active physiological movements of the ankle joint under weight-bearing activity could increase ankle dorsiflexion ROM, while changed ankle mechanics could alter muscle activities ${ }^{11)}$. Therefore, regaining of ankle dorsiflexion ROM by modified MWM using tape could change the flexibility of the GCM muscle; enhanced muscle flexibility, in turn, could allow more efficient movements, which might increase the EMG activity of the GCM. Incline walking with the modified MWM using tape involves active physiological motion, and repetitive motion for 5 minutes could change GCM muscle activity. We think that incline walking with the modified MWM using tape could be applied for effects similar to those of dynamic stretching, which consists of performing movements ${ }^{12)}$.

We observed that there was decreased EMG activity of the TA muscle between heel strike and heel off during inclined walking with modified MWM using tape compared with that without using tape. At least 10 degrees of ankle dorsiflexion is needed during the stance phase of the gait cycle; this contributes to forward body movement for normal walking. The TA muscle is active at heel strike and continues until the loading response; initially, eccentric activity and concentric activity in the second half of the loading response move the tibia forward ${ }^{1}$. To compensate 
for the limited ankle dorsiflexion ROM, the activity of the TA muscle may increase during the early stance phase ${ }^{13)}$. Incline walking with the modified MWM using tape could be helpful to in increasing ankle dorsiflexion ROM, which may contribute to controlling the compensatory strategy and preventing pronation, thereby resulting in decreased activity of the TA muscle.

The EMG activities in both the GCM and TA muscles did not change between heel off and toe off in this study. We focused on the mean EMG activity of the GCM prior to the pre-swing phase because plantarflexion primarily arises from the recoil of the tendon, and actually, the GCM seldom moves between heel off and toe off ${ }^{1)}$. Our data were obtained from a small sample of only young women, so it is difficult to standardize the change in the activities of the lower extremity muscles. We examined healthy people with limited ankle dorsiflexion with no pathological diseases. Further study with a larger number of subjects should be performed to analyze the kinetics and kinematics during incline walking in a synchronous fashion. In addition, we need to investigate the long-term effect of modified MWM using tape through various exercise frequencies. We concluded that incline walking with modified MWM using tape increased the EMG activity of the medial GCM muscle and decreased the activity of the TA muscle between heel strike and heel off. Therefore, our results suggest that modified MWM using tape during incline walking could be an effective intervention for altering the EMG activities of medial GCM and TA muscle in people with limited ankle dorsiflexion.

\section{ACKNOWLEDGEMENT}

This work was supported by the 2012 Inje University re- search grant.

\section{REFERENCES}

1) Perry J, Burnfield JM: Gait analysis: normal and pathological function, 2nd ed. Thorofare: NJ Slack Inc., 2010.

2) Kaufman KR, Brodine SK, Shaffer RA, et al.: The effect of foot structure and range of motion on musculoskeletal overuse injuries. Am J Sports Med, 1999, 27: 585-593. [Medline]

3) McIntosh AS, Beatty KT, Dwan LN, et al.: Gait dynamics on an inclined walkway. J Biomech, 2006, 39: 2491-2502. [Medline] [CrossRef]

4) Wikstrom EA, Hubbard TJ: Talar positional fault in persons with chronic ankle instability. Arch Phys Med Rehabil, 2010, 91: 1267-1271. [Medline] [CrossRef]

5) Hoch MC, McKeon PO: Joint mobilization improves spatiotemporal postural control and range of motion in those with chronic ankle instability. J Orthop Res, 2011, 29: 326-332. [Medline] [CrossRef]

6) Johanson MA, Cuda BJ, Koontz JE, et al.: Effect of stretching on ankle and knee angles and gastrocnemius activity during the stance phase of gait. J Sport Rehabil, 2009, 18: 521-534. [Medline]

7) Mulligan BR: Mobilisations with movement (MWM'S). J Man Mani Ther, 1993, 1: 154-156.

8) Sahrmann SA: Movement system impairment syndromes of the extremities, cervical and thoracic spines. St. Louis: Mosby, 2011.

9) Phadke CP: Immediate effects of a single inclined treadmill walking session on level ground walking in individuals after stroke. Am J Phys Med Rehabil, 2012, 91: 337-345. [Medline] [CrossRef]

10) Kendall FP, McCreary EK, Provance PG: Muscles: testing and function with posture and pain, 5th ed. Baltimore: Williams and Wilkins, 2005.

11) Norkin CC, Levangie PK: Joint structure and function: a comprehensive analysis, 4th ed. Philadelphia: FA Davis, 2001.

12) Cook C: Orthopedic manual therapy: an evidence-based approach. Pearson Prentice Hall, 2007.

13) Murphy DR: Dynamic range of motion training: an alternative to static stretching. Chiropr Sports Med, 1994, 8: 59-66. 